\title{
Difficulties in teaching electromagnetism: an eight year experience at Pierre and Marie Curie University
}

\author{
H. Roussel, M. Hélier \\ L2E, Laboratoire d'Électronique et Électromagnétisme \\ UPMC Univ Paris 6, UR2, L2E, BC 252, 4 place Jussieu F-75005 Paris, France \\ *corresponding author, E-mail: helene.roussel @upmc. fr
}

\begin{abstract}
We will begin by outlining the details of how electromagnetics is taught in the electrical engineering program offered at Pierre and Marie Curie University (a French example) from the undergraduate to the Masters degree levels. We will also discuss how our methods are and should be adapted to teach wave propagation without discouraging students.
\end{abstract}

\section{Introduction}

Teaching electromagnetics in France is an increasingly challenging task. There are several reasons for that. First, many students are tempted to choose Shannon over Maxwell due to the perception that they have of digital devices, ignoring the fact that high data rate systems are electromagnetic ones. Secondly, students feel that electromagnetics is a difficult and demanding subject, as opposed to digital systems design, for instance, which is believed to be easier to grasp and more rewarding. Finally, because of a recent phenomenon of de-industrialization in Europe as well as the social downgrading of technical jobs in companies compared with the importance given to positions related to leading projects or financial management; some students still tempted by an academic career in sciences, prefer to turn to system level activities and are concerned about becoming too specialized as RF engineers. This disinterest is paradoxical as our society deeply depends on electromagnetic systems (mobile phone networks, communication networks, computer system operating now in the microwave band, transportation, even the controversial high frequency trading of the market...). Nevertheless, in other countries, especially in Asia, electromagnetics studies are still appealing to students [1] and when they study in France, their enthusiasm does not wane and they are not discouraged by the level of mathematical skills required. Moreover, many of these students are certain to have the chance in the future to take part in the design and production of RF devices on an industrial scale due to the expansion of many Asian countries and the high international demand for such products. A good summary of the teaching methods of electromagnetism around the world can be found in [2]. This interesting article, published in 1990, shows that electromagnetic courses offered during the Electronic Engineering (EE)
Undergraduate program are comparable in each country and that students' interest in ElectroMagnetism (EM) is continuously declining. During the second year, students are first introduced to static and magnetic fields during a basic EM course; the course on electromagnetic fields (Maxwell's equations) is often given in the third year. Furthermore in all countries, teaching electromagnetism should be combined with teaching mathematics, such as linear algebra, vector calculus, integral calculus and the study of complex variable functions. We believe that this is the main reason why students drop out of courses related to electromagnetism: there is a conflict with mathematics, especially vector calculus and three dimensional representations that cannot be ignored. Another reason is the growing interest for new technologies mostly in the digital domain which are not identified as being linked to electromagnetism courses.

Many initiatives however have been implemented to counter this decline. Some are based on technology (virtual experiments [3] or real ones [4]), some are based more on pedagogy [5] and some on a mixed approach [6]. One of the common goals of these approaches is to allow students to visualize and and give concrete expression to the abstract concepts of electromagnetics. A well known example is the Center of Excellence for Multimedia Education and Technology (CAEME) initiative started in 1991 at the University of Utah under the supervision of Professor Magdy Iskander [7]. This initiative was based on dedicated multimedia software packages that allowed students to learn interactively and perform simulations of electromagnetic experiments. Another interesting program is the Technology Enabled Active Learning (TEAL) approach developed at MIT since 2000 [8]. The idea is here to replace passive learning (traditional lectures) among large classes (500-600 students) by active learning and the use of educational technology applied to smaller groups of students (120 students). Students following the program could carry out desktop experiments, visualize and simulate several electromagnetic phenomena and have Web access to assignments. The interesting and original part of this, is that the effectiveness of such an approach has been carefully assessed in terms of what students retain by comparing the results obtained through pre-tests, post-tests and surveys with two groups of students: the experimental group enrolled in the TEAL pro- 
gram and the control group attending traditional lectures. The experimental group retained significantly more and improved their conceptual understanding of electromagnetics. Even if an enhanced perception of abstract concepts using hands-on activities, visual effects and simulation software is not enough to improve learning, it seems to help students when associated with a well-structured course. Moreover, in terms of this experience, it should be noted that the TEAL approach was applied to smaller classes than the traditional one, and seems to also benefit from better student monitoring.

We focus on the period 2004-2012 because in 2004 the French university system was completely reworked to harmonize our courses with other European countries. This reform has facilitated exchanges between European universities and with universities in North America. At University Pierre and Marie Curie, the number of students enrolled in science courses is around twenty thousand. Approximately five percent of these students follow one sequence (Undergraduate or postgraduate program) in our department (Electronic Engineering).

\section{Courses related to electromagnetism in Electronic Engineering Undergraduate and master programs at the University Pierre and Marie Curie (UPMC)}

During the Undergraduate cycle [9], two courses related to electromagnetism are mandatory for students registered in the EE program. The first, Electrostatics and Magnetism: Fundamentals and Applications, is offered during the fourth semester for twelve weeks with two hours of lectures and tutorials per week and twelve hours of practical training. This gives a total of sixty hours. This course concerns about one hundred students and is followed by all the students enrolled in our department. This first contact with electrostatics is often the most difficult because it involves new mathematical concepts such as vector operators. Every year we change the course to improve this essential step. This is done by trying to make it more concrete and more accessible by facilitating the understanding of physical phenomena through simple examples. To ensure that these concepts are property acquired by our students, we are proposing this year to set up a project for the second semester. This project consists of a questionnaire similar to the Brief Electricity and Magnetism Assessment (BEMA) done by students at the beginning of the semester and end of the semester to measure their initial and final grasp of concepts. This is a standard procedure in many countries, particularly the United States [10]. This project involves the maximum number of students to include students from different tracks at UPMC, general physics, applied physics and EE at the same level (second year). The average success rate for this course is close to forty five percent but we try to increase it to make it more appealing.

The second course takes place during the last semester of the EE undergraduate program and is called, Radiation and Propagation, it follows the same framework as the former course. Using a more punchy course title: Application, Radiation and Propagation helped students see the immediate relevance and applicability of these courses. Following a poll to determine what the students learned and where their interests lie, we found that they continue to express regret about not having enough practical courses. Indeed, given the cost of equipment used to develop practical work on electromagnetism and the time needed to learn how to use this material, we limited this teaching in a four-hour session. This session combines measurements of small dipoles with or without reflector/ one or more director and simulations of wire antennas (see figure 1) with the free Numerical Electromagnetic Code (NEC-4) based on the method of moments. During this session, students compare measurements with simulations and analytical solutions given in classroom. The number of students involved in this course is the same as the first because it is a companion one. The success rate of this course is slightly higher than the first one, around fifty five percent. This is not enough for us but be aware that for about two thirds of our students, electromagnetics theory stops at the end of the Undergraduate cycle.

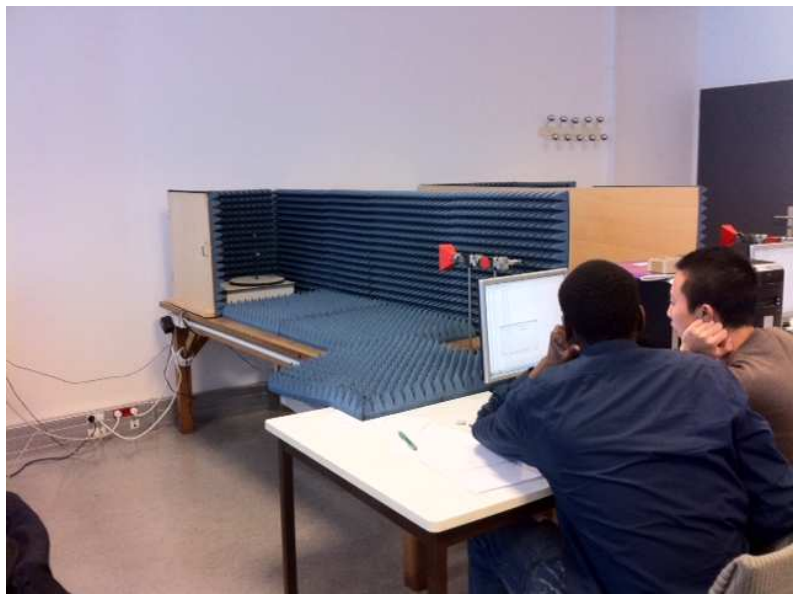

Figure 1: measurements facilities

The postgraduate program [11] is easier to put together. Indeed, when students get to the first level of the Master's degree they can choose among five specialities. Two of these concern EM: the Communication Systems program and, to a lesser extent, the Sensors, Measurements and Instrumentation program. Teaching electromagnetism at this level is easier because students have opted to pursue this field of study and are more receptive to electromagnetic waves; nonetheless the topic remains theoretical and complex for most students. Several courses are offered to the students. The first is called Mathematics for Communication Systems and takes place during the first semester of the first year. It provides the students with all of the mathematical tools (generalized functions, Green's functions, Hilbert's spaces, probabilities) required to understand 
theory and recent concepts in electromagnetics. The next courses are provided during the second semester of the first year and deal with Electromagnetic Compatibility (an introduction to EMC), Antennas, Transmissions Lines and Guiding Structures.

During the first semester of the second year of the Communication Systems program, complementary courses allow students to deepen their knowledge in electromagnetics, depending on the program they have chosen. Students registered in the Electronic On-Board Systems program follow an Advanced Electromagnetic Compatibility course with a final exam requiring the analysis and synthesis of a paper. This course is boosted by several talks given by experts from the transportation industry (Dassault Aviation, Eurostar and Renault) who give practical examples of on-board systems and explain electromagnetic compatibility issues with regard to industrial equipment. Students registered in the RF and Microwaves System program will attend an Advanced Antennas course and an Advanced Microwave Devices course as well. The students in both programs take courses on Mobile Communication Systems. The second semester of the second year is devoted to a sixmonth internship in a company or laboratory. Some of these companies, like Thales, Orange, Bouygues Telecom, Ericsson are involved in the design or use of microwave systems, others like PSA Peugeot Citroën, Renault, Safran or Alstom develop, produce and market vehicles (cars, trains) or engines for airplanes in which high frequency devices are integrated or in which high frequency phenomena may be encountered (lightning for instance).

\section{Some proposals to increase the attractiveness of electromagnetism}

In order to encourage students to choose Maxwell instead of Shannon, one solution would be to decouple physical understanding from mathematics by using numerical packages such as Matlab, Maple and Mathematica, as suggested in [12] and [13]. In our department we combine the use of these computer algebra systems with continuous development of new practical courses (antenna measurements combined with complex computation done using EM tools, EMC measurements, guided waves measurements, Radar simulation, etc.) as well as new applicable tutorials. Confucius said "I hear, I forget. I see, I remember. I do, I understand." This saying is particularly pertinent in terms of abstract teaching. The best way to understand the mechanisms involved in the interaction of a wave with its environment is to perform EM measurements for various configurations rather than to develop the equations which govern the propagation in the medium considered.

We give you two examples of practical courses offered at UPMC during EE graduate program. The first concerns EMC measurements. In our department we have designed a metallic box (copper) with or without a small opening on one side. The dimensions of the box are determined so that several resonance frequencies appear in measurements frequency band $(300 \mathrm{MHz}-1 \mathrm{GHz})$. The experiment is carried out in a Faraday cage (see figure 2). In the box we introduce a field sensor for measuring illumination at different frequencies and configurations of the box. These measurements allow us to highlight the effects of leakage through the side slots of the copper box.

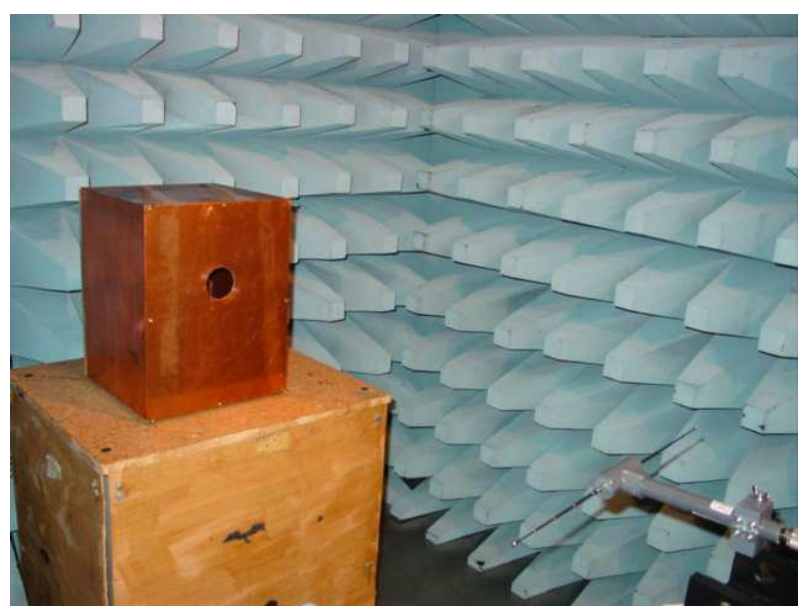

Figure 2: EMC measurements

For the course entitled "Radar and Location", we benefit from the Learning and Teaching version of the software MOCEM (MOCEM LT), an "all in one" tool to simulate SAR image for different configurations (see figure 3). MOCEM LT has been offered by DGA MI to UMPC for teaching purposes on radar and synthetic aperture radar imagery. The LT version of MOCEM is a limited version with less functionality than the original software MOCEM V3 developed by Alyotech under contract and specifications of the DGA (French Defense Agency) [14]. MOCEM principles are based on Geometrical Optics/Physical Optics principes but with a kind of shorcut in the computation using a estimation the EM level that results of the SAR intregration process. Two phenomena are considered, the diffuse effect resulting from many incoherent scatterers in the image resolution cell and the coherent response coming from reflective surfaces (ground and dielectric or metallic plates). The advantage of this software is that we can easily illustrate many courses (radar, EM propagation) by analyzing the effects observed in the $3 \mathrm{D}$ view of the main mechanisms (figure 4) or by observing the synthetic image for different radar configurations (figure 5).

These experiments are very popular with students but obviously go hand-in-hand with lectures that will reinforce the observations made during the experiments.

\section{How to improve the content of our courses?}

A good way to continually improve the content of courses for students is to interview them to assess if all of the concepts taught are assimilated. In our department this year, we purchased a device called 


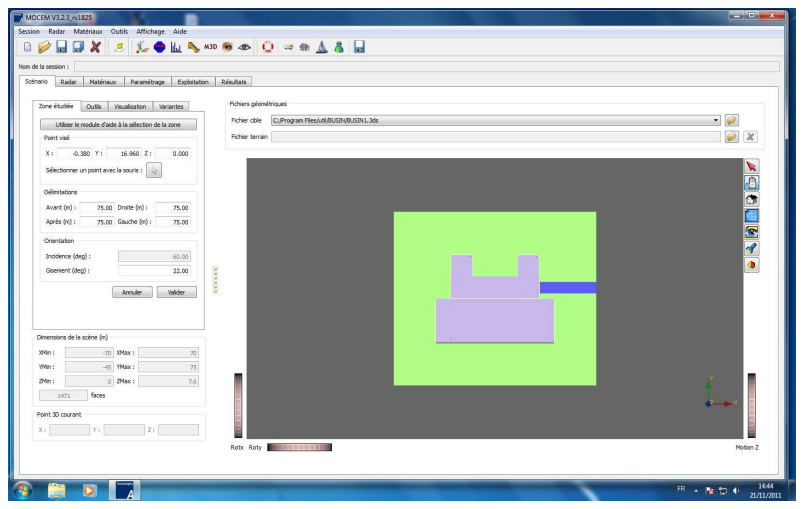

Figure 3: One of the proposed configuration in MOCEMLT: industrial building

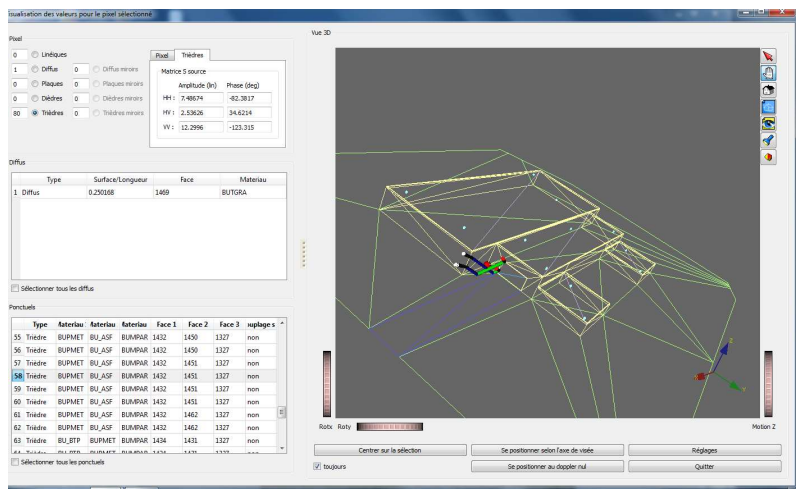

Figure 4: 3D view of the main mechanisms
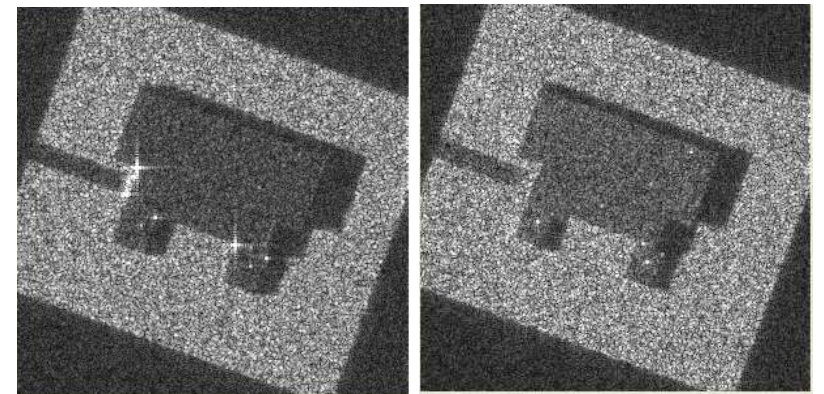

Figure 5: synthetic image for two incident angles: $60^{\circ}$ and $40^{\circ}$

TurningPoint $\mathrm{R}$ AnyWhere ${ }^{\mathrm{TM}}$ that allows students to take part in lectures by submitting responses to interactive questions using a ResponseCard ${ }^{\mathrm{TM}}$ keypad. The advantage of this tool is that it allows us to query instantly and anonymously some sixty students. In this way, we can almost immediately adapt our course if we see that concepts are not being well assimilated. I give you in figure 6, an example of output that can be achieved with this device. For this example, the student group interviewed is composed of thirty five participants. This initiative began at UPMC in 2011 and is comparable to that proposed in [15]. Thus, we have not enough experience to apreciated the benefits but if you look at the results presented in [15], we can expect a significant increase in the acquisition of basic concepts of electrostatics, magnetostatics and electromagnetism.

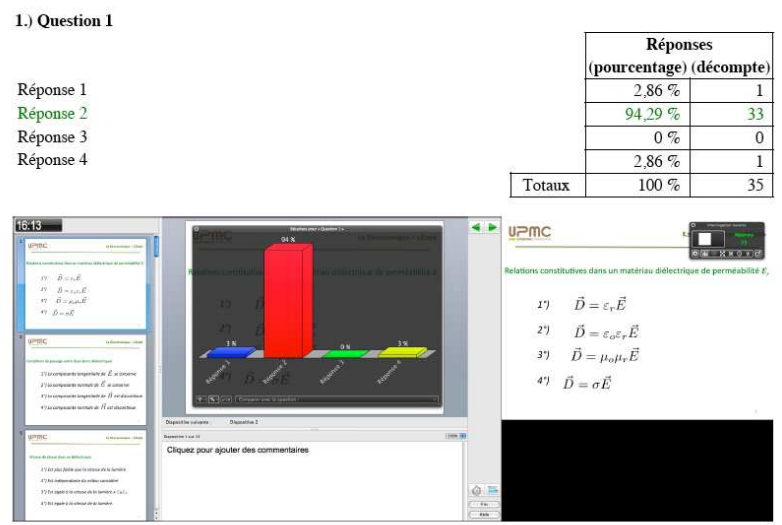

Figure 6: TurningPoint $\AA$ AnyWhere ${ }^{\mathrm{TM}}$ device output

\section{Conclusion}

The teaching of electromagnetism in electrical engineering courses must be tailored to an audience which is more and more reluctant to adhere to abstract disciplines. In our opinion, the best solution for making EM courses more attractive is to offer as early as the first year, practical applications and intensively use of mathematical computer-aided teaching tools to overcome, at the beginning, the difficulties linked to mathematics. Finally, after the first two years of the Undergraduate program, it is necessary to return to these mathematical concepts through further EM courses in the first year of the Master's program.

\section{Acknowledgement}

The authors would like to thank all their colleagues teaching electromagnetics at Pierre and Marie Curie University, involved in the undergraduate program (bachelor), their colleagues teaching in the "Communication Systems" program (master, postgraduate program) and DGA for its contribution.

\section{References}

[1] X. M. Zhang, S. L. Zheng, Y. Du, X. F. Ye and K. S. Chen, Perspective of Electromagnetics Education, Progress in Electromagnetics Research Symposium Proceedings, Xi'an, China, March 22-26, 2010.

[2] F.-J. Rosenbaum, Teaching Electromagnetics Around the World : A Survey, IEEE Trans. on Education, Vol. 33: 22-34, 1990.

[3] L. Sevgi, A New Electromagnetic Engineering Program and Teaching Via Virtual Tools, Progress In Electromagnetics Research B, Vol. 6, 205-224, 2008. 
[4] M. Lumori, E. Kim, Engaging Students in Applied Electromagnetics at the University of San Diego, IEEE Transactions on Education, Vol. 53, No.3, August 2010.

[5] J. Leppävirta, H. Kettunen, A. Sihvola, Complex Problem Exercices in Developing Engineering Student's Conceptual and Procedural Knowledge of Electromagnetics, IEEE Transactions on Education, Vol. 54, No.1, February 2011.

[6] J. Bunting, R. Cheville, VECTOR: A Hands-On Approach That Makes Electromagnetics Relevant to Students, IEEE Transactions on Education, Vol. 52, No.3, August 2009.

[7] O. de los Santos Vidal, R. Jameson, M. Iskander, Interaction and Simulation-Based Multimedia Modules for Electromagnetics Education, FIE '96 Proceedings, 1996.

[8] Y.-J. Dori, E. Hult, L. Breslow and J. Belcher, How Much Have They Retained? Making Unseen Concepts Seen in a Freshman Electromagnetism Course at MIT, Journal of Science Education and Technology, Vol. 16, No. 4, August 2007.

[9] http://www.licence.elec.upmc.fr.

[10] L. Ding, R. Chabay, B. Sherwood, R. Beichner, Evaluating an electricity and magnetism assessment tool: Brief electricity and magnetism assessment, Physical review special Topics- Physics education research 2, 2006.

[11] http://www.upmc.fr/fr/formations/departements.html.

[12] D. Ioan, I. Munteanu, Symbolic computation with Maple V for undergraduate electromagnetics education, IEEE Trans. on Education, Vol. 44:217, 2001.

[13] A. Norman, M. Mani, A new approach in teaching electromagnetism: how to teach EM to all levels from freshman to graduate and advanced-level students, Proc. American Society for engineering education annual Conference, 2003.

[14] C. Cochin, P. Pouliguen, B. Delahaye, D. Le Hellard, P. Gosselin, F. Aubineau, MOCEM -An 'all in one' tool to simulate SAR image, Synthetic Aperture Radar (EUSAR), 2008.

[15] A. Fagen, C. Crouch, E. Mazur Peer instruction: Results from a range of classrooms, The Physics Teacher, Vol 40, April 2002. 